\title{
MOMENTARILY EXCESSIVE CONSTRUCTION AS THE BASIS FOR PROTOADAPTATION
}

\author{
Carl Gans \\ Division of Biological Sciences, The University of Michigan, Ann Arbor, Michigan 48109
}

Received February 1, 1977. Revised May 23, 1978

The concept that all individual organisms are perfectly matched to their environment is clearly a false idealization. Actually, for most individual organisms the structural and physiological capacities are likely to be excessive ${ }^{1}$ for the needs of any particular moment. In the present context, "excessive construction" refers to excess capacity, but only in terms of the state of a single character of a particular individual at a particular instant in time. This concept has no implications about the perfection of matching of any population of a species to the particular biotope it currently inhabits. Obviously, natural selection does not "look" just at an organism's momentary utilization of each aspect of the phenotype, but at the requirements imposed on all phenotypic aspects of an individual throughout its life span.

The "excess" does represent a paradox. Why should phenotypes be overdesigned? Statements that such overdesign represents a "factor of safety" (cf. Kummer, 1959) hardly explain its origin. In any case they imply technological planning or prescience and should probably be discouraged. Although excessive construction

\footnotetext{
1 Some of the reviewers of this manuscript appear to be a bit unhappy about the term "excessive construction" (which is here used in a sense very different from the term "excessive types" applied by Rensch (1959) to various giant forms). For a while, I was tempted to substitute for "excessive construction" the term "superrogation" which Ernest Williams indicates as one he has been using in lectures. I desist because I believe the term excessive to be descriptive and appropriate. I must stress that while the term may imply increase of the phenotypic aspect under discussion, the phenomenon involves both the "lower" and the "upper" limit of the frequency histogram of a character state of a population.
}

may be paradoxical, it is important because it permits an individual animal degrees of freedom in its behavior and physiology; the excess allows novel behavior and, with this, adaptive shifts. For instance, the excess allows some omnivorous birds to use their relatively slender beaks occasionally to crush small seeds or a tit to use its beak to open milk bottles, in each case without much risk of fracture. The ribs and vertebrae of a bull snake allow this snake on occasion to eat hardshelled bird eggs, breaking the shells within the intestinal canal by pushing the body against the walls of a tunnel or a rock, without a risk of fracturing bones or bruising soft tissues. Accordingly the origin and importance of "excessive constructions" may deserve a brief reemphasis.

\section{Diversity of Individual Phenotype AND OF SELECTION}

The expression of most characteristics of any organism is in some way affected by environmental conditions; hence the phenotypes of even genetically identical individuals ordinarily vary because of minor differences in the environmental conditions they encountered during development. Additional intrapopulational variability can be ascribed to such factors as mutation, recombination, gene flow, genetic drift, and various polymorphisms maintained by selection. All of these factors explain why the observed frequency distributions of character states rarely show sharp peaks, but rather some central tendency, with relatively few individuals falling in the tails of the curves. It has also been argued (Gans, 1974; Wainwright et al., 1976) that the selection against the 
condition represented by the tails represents a balance between the benefit of a modification permitting survival under extreme conditions and the cost of constructing and maintaining such extreme phenotypes at other times.

Individuals have the same probability of encountering an event that will involve a high selective advantage for a given size and level (magnitude or qualitative state) of a particular phenotypic feature, but only some of them need to encounter it. In simple terms, this means that there is a distribution of stressful events (a probability distribution of selection pressures) which act on the population of organisms which exhibits in turn a frequency distribution of phenotypes. The probability that a certain feature will permit an individual organism to survive, then reflects both the phenotypic level of the particular feature in the individual, as well as the timing and magnitude of the environmental demands on the feature that the particular individual happens to encounter. Naturally the individual phenotype falls within the populational range of phenotypes and the particular momentary environmental demand falls within the range of enviromental demands encountered by the population as a whole.

Consequently, we may at any time observe up to nine classes of events representing a simple three by three set. The range of demands imposed by the environment may be subdivided into three categories $(i=$ high, $i i=$ medium, $i i i=$ minimal) as may be the kinds of phenotypes $(1=$ maximal, $2=$ average, $3=$ minimal). We can see that three classes of the possible events $(i, 2 ; i, 3 ; i i, 3)$ lead to failure, three other classes $(i, 1 ; i i, 2$; iii, 3) show survival because the phenotype is capable of meeting momentary demand, and that three classes (ii, $1 ; i i i, 1$; $i i i, 2)$ show survival because the pheno- types incorporate an excess of capacity. This description deals only with the possibility of surviving the particular event; it does not consider other advantages or costs of maintaining the "maximal," "medium" and "minimal" phenotypes, the fractions of the population belonging to each category, nor the fraction of events classified as "high demand," "medium demand" or "minimal demand." Indeed it does not indicate whether these selective events represent a steady state or vary with time.

\section{REASONS FOR THE EXCESS}

It is a common observation that this scheme matches actual conditions. Individual animals often bear structures that seem to incorporate more (or less) material or have greater (or lesser) physiological capacity than that needed (or permitted) to let an individual animal survive at that particular instant in time. The birds and snakes cited in the introduction offer examples of this phenomenon. Indeed, a common finding is that structural elements in animals may carry heavier loads than those ordinarily observed, muscles can exert greater forces than normally required and that other aspects of the phenotype permit functions rarely or never imposed. Why are there such excessive phenotypes?

The extra capacity which is unneeded at the particular instant may first of all be due to compensation for developmental uncertainty. Developmental uncertainty only means that environmental and genetic variability will produce a population with a characteristic frequency distribution of any phenotypic aspect. If selection requires, for example, that all individuals of a population must be less than $2 \mathrm{~m} \mathrm{(X}$ in Fig. 1) high, or more than $30 \mathrm{~cm}$ wide (assume the polarity of the abscissa for this character state reversed in Fig. 1), this

FIG. 1. Distributions and limiting maximum size $(X)$ to show the uncertainty of specifying a limiting condition. Example (c) assumes reduction of size below $X$ does not increase advantage; example (d) assumes the upper limit position has changed for genotype $\mathrm{A}^{\prime \prime}$; example (e) genotype $\mathrm{A}^{\prime \prime}$ is small with $Y$ being a lower limit below which disadvantage increases (being a mirror image set of conditions with respect to $X$ ). 


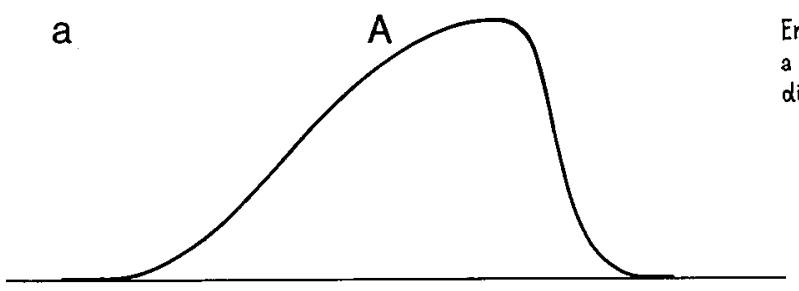

Environmental diversity causes a genotype $A$ to be read into 2 distribution of phenotypes

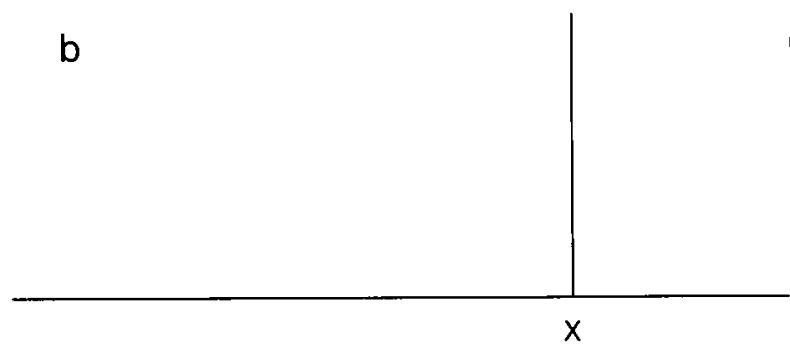

It is advantageous to be smaller than $X$
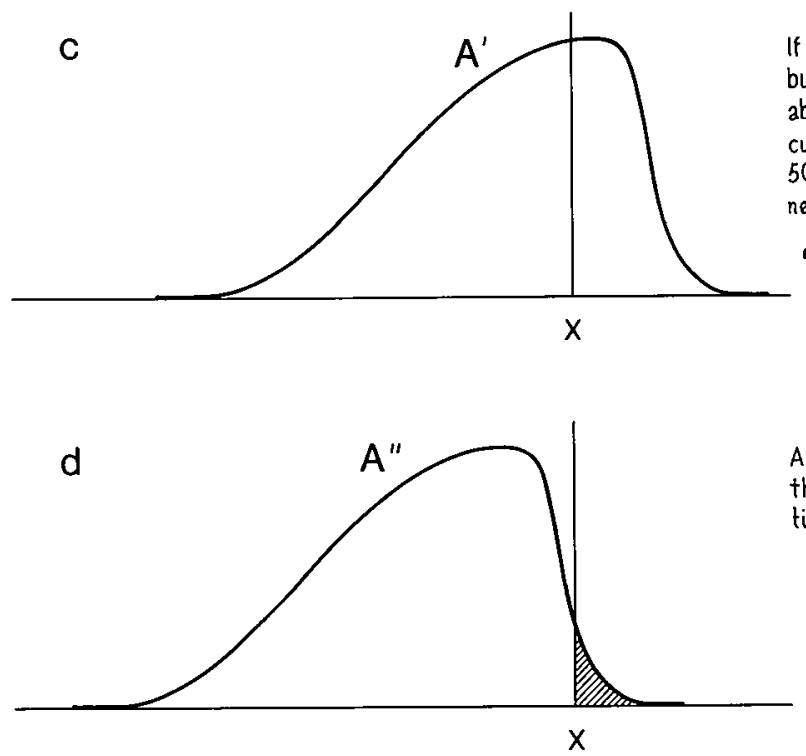

If the mean phenotupe produced by genotype $A^{\prime}$ equals $X$, then about $50 \%$ of the population incurs a disadvantage land about $50 \%$ of the populations is unnecessarily small).

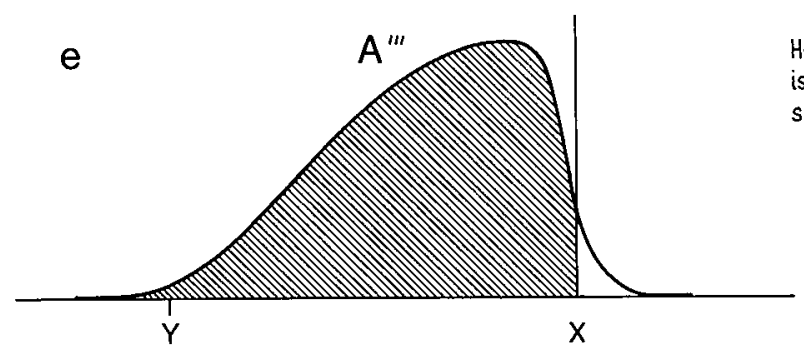

A shift to genotype A" means that tess than $5 \%$ of the population incurs the disadvantage

However $95 \%$ of the population is now unnecessarily ("excessively" small. 
would assure that almost all individuals or a successful form would likely be shorter or wider than these dimensions. This is an old idea that was well characterized by Dobzhansky (1951:106) in his discussion of "reaction norms," i.e. of genotypes buffered to produce an acceptable range of phenotypes when development took place under any of the conditions "expected" by the species.

[I do not, for the moment, choose to deal in detail with the effects of pleiotropy or of phenotypic strategies, such as homeostasis versus polymorphism (Levins, 1968). These aspects generally affect the relative frequency, but not the nature of the nine classes of events actually observed.]

Another and more important cause for excessive capacity is the occurrence of rare events, which in the present context are those that are uncommon, but still important to the animal. The frequency and duration of utilization for any biological feature of an animal obviously vary. Furthermore, it should be obvious that not all events during which the feature is utilized are equally critical to the survival of the organism. However, some rare events are likely to have an extreme effect on the shaping of the phenotype. For instance, some amphisbaenians and skinks generally burrow in finely and evenly particulate soils and sands (Gans, 1974). In such uniform media the stress distribution within their skulls would remain uniform and its level low. However, burrowers occasionally encounter nonhomogeneous substrates and may contact sharp-edged objects at an angle, which results in asymmetric and local stress concentrations. These stresses would produce failure unless both shield and skull are generally reinforced to carry four or five times the "normal" loads in order to cope with such occasional events. While it has been impossible to quantify the observations, it also appears that restructuring and reinforcement of the skull limits prey-killing ability. Thus the skull is apparently strengthened in spite of a major cost. One could consider such encounters uncom- mon events, only predictable statistically (i.e. some individuals may never hit a rock); the selection pressures that maintain head shape and cranial reinforcement should differ among soil types.

A spectacular example characterizes the class of directly predictable events that are rare only in terms of the total time they occupy in the life span of the animal. Adult Arican crocodiles (Crocodylus niloticus) reach weights of $750 \mathrm{~kg}$ and can crush cattle bones with their massive jaws. Yet these large animals have surprisingly fine control of the mandibular musculature and can pick up and manipulate small objects between their jaws. This capacity apparently achieves its selective advantage during the reproductive season as the parents then dig up the nest, pick up the tiny (100 to $125 \mathrm{~g}$ ) hatchlings between the enormous jaws, and carry them to the water in their buccal cavity. The motor control would seem unnecessarily fine were one only to study the action of the jaws during feeding; however, reduction of motor control will immediately and adversely affect the animal's fitness (Pooley and Gans, 1976). The difficulty of analysis in an actual case is seen by the observation that even though the female normally excavates the eggs, the male is capable of doing so-though we lack records of this behavior in the wild. Certainly the fine motor control would appear unnecessary during the life span of most males (unless selection has indeed been for an aspect of feeding that we have been unable to note).

Many other examples of such rare but predictable events may be cited. For instance, pine trees have adaptations for fires that may occur less frequently than once a decade. Some desert plants show reproductive systems that maximize the benefits of occasional rainstorms or flash floods.

\section{EXCESSIVE CONSTRUCTION AND Protoadaptation}

Small gill-breathing fishes can pick up air bubbles through the water surface when the dissolved oxygen content drops 
and hold these bubbles within the gill chambers without rupturing the gill tissues. Generalized round-headed amphisbaenians, that normally tunnel straight through the soil, can bend or twist the head on the body axis. These movements exert forces on the sides and end of the tunnel without causing the supportive tissues of the animal to fail in bending. In each case the organism's structures support the unusual demands, though the organism engages in a variant or unusual behavior. The "excess" need not involve the avoidance of failure, but might instead facilitate behavior in other ways. For instance, the crocodilian motor control, mentioned earlier as an example of "momentarily excessive construction," apparently allows crocodiles to perform fine manipulations during feeding, such as unwrapping human bodies from multiple layers of cloth (A. C. Pooley, pers. comm.).

In most cases, the divergent behavior may not contribute significantly to the animal's fitness. However, such incidental behavioral variants may, on occasion, allow the animal to exploit a new resource or may improve the use of an old one. The particular behavior will then produce a selective advantage for the individual performing it. With this it will increase the probability that the behavior could occur in subsequent generations. Secondarily there will then be potential selection for enhancement of the morphological and physiological characteristics serving as a basis for the behavior. Consequently, one may argue that the excessive capacity of the phenotype incorporates protoadaptations ${ }^{2}$ for the new behavior. Even though a particular phenotype may have been se-

\footnotetext{
${ }^{2}$ The term preadaptation, often used in this context is, as documented by Simpson (1953), flawed by its earlier and prolonged association with the concept of orthogenesis. Unfortunately Simpson also used the term in the present sense. Consequently, I propose the use of the term protoadaptations for those biological features that, while they may have been developed initially in association with one biological role, allow the organism also to perform a different and generally unrelated role.
}

lected for one set of biological roles, the intrinsic overconstruction allowed the behavior to shift to a new biological role. In this sense then the phenotype or "structure" precedes the biological role or function (= behavior in this context). If such a utilization of a particular phenotype is advantageous to the individuals involved, this ability establishes an immediate selective advantage for the further modification of the phenotype.

An example of an apparently behavioral shift that may have facilitated extension of habitat and thus defined a wider environmental niche for a species is furnished by the adaptation of the snake Nerodia sipedon to the fresh and brackish water locations this species inhabits. In neither locality can the species use brackish water for drinking. Members of both populations obtain enough water from their food to survive without free water and both apparently have a skin that prevents ion entry (or loss) or significant osmotic water loss to the brackish water even when wetted for long periods.

The ancestral populations (and almost all other members of the genus Nerodia) presumably were associated with fresh water biotopes, but are likely to have had the capacity to taste the difference between fresh and salt water. The only real difference between these ancestral populations would then have been the behavioral one of recognizing and not drinking brackish water; behavior now observed in the marsh form. When the fresh water form is offered brackish water, it supposedly drinks it and succumbs. Apparently the (now presumably innate) behavioral utilization of once "excessive" capacity of the integument to prevent osmotic inflow of salt, as well as the capacity to taste saltiness, allowed some populations of this species to extend their biotope (Pettus, 1958; but see Dunson, 1977).

A similar behavioral (perhaps sensory) adaptation utilized in the opposite direction is seen in the marine seasnake Laticauda colubrina. This snake, though predominantly marine, can recognize and will drink from a dish of fresh water floating 
atop the salt water that fills the aquarium in which it is swimming (pers. obs.). The snake approaches the dish and drinks its contents even though it is never observed to drink the salt water. The discriminatory capacity may have occasional adaptive value, as the species ascends rivers and occurs in semiterrestrial situations (Saint Girons, 1964).

Two additional examples derive from parrots. The New Zealand Kea (Nestor notabilis) has shifted from a vegetarian and insectivorous diet to killing and feeding on sheep and goats (Oliver, 1955). The curved bill permits them to rip open the tough skin. In Western Australia, the whitetailed black cockatoo (Calyptorhinus baudinii latirostris, but not the nominal race) have invaded the introduced pine plantations and can extract the seeds from the tough cones of Pinus pinaster and radiata (Perry, 1948; Saunders, 1974). Observations suggest that this utilization of a "new" resource has markedly increased the flock size and perhaps the overall number of individuals of this species.

Swimming may be considered a potential behavioral adaptation that might one day expand the habitat of certain agamid lizards (it may have already done so outside of Sri Lanka). Populations of the bloodsucker lizard, Calotes sp., inhabit Sri Lanka, an island on which there has historically been no standing water (Gans, 1978). Like many lizards Calotes can swim by lateral undulations of the trunk and have a specific gravity sufficiently low so that their head remains emergent and they can breathe while afloat. However, (1) they apparently lack the capacity to stay in an undulant rather than an alternating quadrupedal swimming movement, and after they shift to the latter they tire rapidly and tend to swim more slowly; (2) though easily able to float with the head above water, they lack the behavior of breathing when only their nostrils are in air. Consequently, they soon inhale water and sink to the bottom where they drown unless rescued. The swimming of Calotes is obviously not a "normal" part of its behavioral repertoire, but the "miss- ing" behavior could, in other regions, make an aspect of the biotope available. One would then expect to see further structural development, culminating in specializations such as those shown by some iguanid lizards of very similar habitus that swim well, such as the iguanid genus Basiliscus (Laerm, 1973), as well as Iguana, Amblyrhynchus, and Anolis aquaticus.

\section{SUMMARY}

Most aspects of phenotypes will, at any moment of an individual's life, be capable of fulfilling demands much greater than those routinely encountered. This capacity gives members of a population of organisms the capacity to modify their behavior. If the new behavior allows the species to enter a new adaptive zone (sensu Simpson, 1953), the direction of selection will change. Those individuals sharing the behavior will have access to a relatively greater amount of resources, hence there will be a tendency to fix the feature genetically, perhaps to increase its magnitude, and certainly to increase the frequency of the formerly "excessive" construction in the population. In short, the phenotypic level once "excessive" in the population is no longer so and the new, stabilized mean will give rise to constructions "excessive" relative to the new mode of life history.

\section{ACKNOWLEDGMENTS}

I am grateful to P. F. A. Maderson for the discussions that encouraged me to write this paper and to B. Clark, G. Dalrymple, A. Dhont, H. Gay, A. S. Gaunt, S. L. Gaunt, G. C. Gorniak, R. Huey, H. J. de Jongh, P. F. A. Maderson, R. G. Northcutt, E. C. Olson, T. S. Parsons, P. Regal, H. I. Rosenberg, S. N. Salthe, E. Spiess, R. Sokal, L. Van Valen and R. Wasserzug for diverse and trenchant comments on the several instars of this manuscript. The responsibility for the final version is clearly my own. Supported by NSF DEB 76-19289. 


\section{Literature Cited}

Dobzhansky, Th. 1951. Genetics and the Origin of Species. Third edition. Columbia Univ. Press, N.Y. ii +364 pp.

Dunson, W. A. 1977. Physiological and behavioral factors leading to the death of fresh-water snakes in sea water. Amer. Soc. Ichth. Herp. Program and Abstracts, Gainesville, Fla.

GaNs, C. 1974. Biomechanics: An Approach to Vertebrate Morphology. J. P. Lippincott, Philadelphia, $x+261$ pp. 1978. Locomotor responses of Calotes to water. J. Bombay Nat. Hist. Soc.

Kummer, B. 1959. Bauprinzipien des Säugerskeletes. Thieme, Stuttgart, $x i+235$ pp.

LAERM, J. 1973. Aquatic bipedalism in the basilisk lizard: the analysis of an adaptive strategy. Am. Midland. Nat. 89(2):314-333.

LeviNS, R. 1968. Evolution in Changing Environments. Princeton Univ. Press, Princeton, N.J. $120 \mathrm{pp}$.
Oliver, W. R. B. 1955. New Zealand Birds. Fine Arts (N.Z.) Ltd., Wellington, N.Z. 661 pp.

PERRY, D. H. 1948. Black cocatoos and pine plantations. West. Aust. Natur. 1(7):133-135.

PetTus, D. 1958. Water relationships in Natrix sipedon. Copia 1958(3):207-211.

Pooley, A. C., AND C. Gans. 1976. The Nile crocodile. Sci. Amer., 234(4):114-124.

Rensch, B. 1959. Evolution Above the Species Level. Columbia Univ. Press, N.Y., xvii + 419 pp.

SaInT Girons, H. 1964. Notes sur l'ecologie et la structure des populations des Laticaudinae (Serpentes, Hydrophidae) en Nouvelle Caledonie. Terre Vie (2): $185-214$.

SAUNDERS, D. A. 1974. Subspeciation in the whitetailed black cockatoos, Calyptorhynchus baudinii, in Western Australia. Aust. Wildl. Res. 1:55-69. Simpson, G. G. 1953. The Major Features of Evolution. Columbia Univ. Press, New York, xx + $434 \mathrm{pp}$.

Wainwright, S. A., W. D. Biggs, J. D. Currey, AND J. M. Gosline. 1975. Mechanical Design in Organisms. Wiley, New York. xii +423 pp. 